\title{
Enhanced recovery program in liver resection surgery: a single center experience
}

\author{
Yuan Ding ${ }^{1,2,3 \#}$, Zhenzhen Gao ${ }^{1,3 \#}$, Zhongquan Sun ${ }^{1,2,3}$, Qiyi Zhang ${ }^{1,2,3}$, Bo Zhou ${ }^{1,2,3}$, Zhiwei Li ${ }^{1,2,3}$, Wenyan \\ Zhang $^{1,3}$, Yuancong Jiang ${ }^{1,3}$, Xiangying Zhang ${ }^{1}$, Guomei Ge ${ }^{1}$, Matteo Ravaioli ${ }^{4}$, Chris Jones ${ }^{5}$, Sheng \\ Yan $^{1,2,3}$, Weilin Wang ${ }^{1,2,3}$; written on behalf of the AME Liver Surgery Collaborative Group
}

${ }^{1}$ Division of Hepatobiliary and Pancreatic Surgery, Department of Surgery, First Affiliated Hospital, School of Medicine, Zhejiang University, Hangzhou 310003, China; ${ }^{2}$ Key Laboratory of Precision Diagnosis and Treatment for Hepatobiliary and Pancreatic Tumor of Zhejiang Province, Hangzhou 310003, China; ${ }^{3}$ Collaborative Innovation Center for Diagnosis Treatment of Infectious Diseases, Hangzhou 310003, China; ${ }^{4}$ Department of General Surgery and Transplantation, S. Orsola and Malpighi Hospital, University of Bologna, Bologna, Italy; ${ }^{5}$ Department of Anaesthesia and Surrey Peri-operative Anaesthesia and Critical Care Collaborative Research Group, Royal Surrey County Hospital NHS Foundation Trust, Guildford, UK

Contributions: (I) Conception and design: W Wang, S Yan, Y Ding; (II) Administrative support: W Wang; (III) Provision of study materials or patients: W Wang, S Yan; (IV) Collection and assembly of data: Y Ding, Z Gao, Z Sun, Q Zhang, B Zhou, Z Li, W Zhang, Y Jiang, X Zhang, G Ge; (V) Data analysis and interpretation: Z Sun, Y Ding, Z Gao; (VI) Manuscript writing: All authors; (VII) Final approval of manuscript: All authors.

\#These authors contributed equally to this work.

Correspondence to: Weilin Wang; Sheng Yan. Division of Hepatobiliary and Pancreatic Surgery, Department of Surgery, First Affiliated Hospital, School of Medicine, Zhejiang University, 79 Qingchun Road, Hangzhou 310003, China. Email: wam@zju.edu.cn; shengyan@zju.edu.cn.

Background: The enhanced recovery after surgery (ERAS) program promotes postoperative recovery. The study aimed to evaluate the effects of the ERAS protocol in hepatectomy.

Methods: The primary outcomes included time to first flatus and length of hospital stay (LOS). Secondary outcomes included time to first passage of stool, time to off-bed activity, pain score, abdominal distension score, postoperative liver function and complications. Outcomes were evaluated between the ERAS group $(n=69)$ and the control group ( $n=232)$. A subanalysis of probiotics use in the ERAS group was also performed. Results: In patients undergoing laparoscopic and open hepatectomy, time to first flatus after surgery was significantly shorter, as well as LOS, in the ERAS group. Lower incidence of post-operative complications was noted in ERAS group. While sub-analysis of probiotics group showed no significance in the primary outcomes.

Conclusions: Implementation of the ERAS protocol for hepatectomy could significantly promote postoperative recovery. Short-term application of probiotics during the perioperative period did not improve the outcomes in the ERAS group.

Keywords: Enhanced recovery after surgery (ERAS); hepatectomy; laparoscopic hepatectomy; probiotics

Submitted Jul 24, 2018. Accepted for publication Aug 16, 2018.

doi: $10.21037 /$ tcr.2018.08.30

View this article at: http://dx.doi.org/10.21037/tcr.2018.08.30

\section{Introduction}

Enhanced recovery after surgery (ERAS) refers to the use of a series of evidence-based perioperative optimization measures to reduce the physiological and psychological traumatic stress response in surgery patients to achieve rapid rehabilitation (1). This concept was first proposed by Kehlet et al. (2) in the 1990s and was initiated as a multimodal approach to promote postoperative recovery. A meta-analysis comprising 38 studies across a range of surgical specialties has demonstrated that ERAS reduced the risk of all complications by $\sim 30.0 \%$ and the duration 
of primary hospital stay by $>1.0$ days (3). ERAS programs have been successfully applied in multiple fields, such as colorectal surgery (4), upper gastrointestinal surgery (5), and genitourinary surgery (6); however, evidence for the effectiveness of ERAS programs in the field of liver surgery, particularly in laparoscopic hepatectomy, remains insufficient.

Previous research on the ERAS program for liver surgery have shown positive effects on promoting postoperative recovery $(7,8)$. A recent meta-analysis of randomized controlled trials concluded that the ERAS program for liver surgery significantly reduced overall morbidity rates, accelerated postoperative recovery, and shortened the primary and total hospital stays without compromising readmission rates (9); however, this meta-analysis was limited by the small sample size. Further studies are required to provide more concrete evidence.

The ERAS protocol in our center recommended the oral intake of nutrients and mobilization in $24 \mathrm{~h}$ after surgery. A nasogastric tube was not routinely used in the ERAS group but, if used, was removed immediately after surgery. After applying the ERAS protocol, complaints of abdominal distension appeared to be more common. Early removal of the nasogastric tube might have been the main cause of this discomfort. Administration of probiotics has been reported to significantly accelerate the return of bowel functions after surgery (10). Thus, application of probiotics during the perioperative period in the ERAS group was adopted to promote the recovery of postoperative gastroenteric function.

The present study was designed to evaluate the outcomes of implementing the ERAS protocol in hepatectomy patients and assess whether application of probiotics during the perioperative period could improve the outcomes of patients following ERAS protocol.

\section{Methods}

\section{Patients}

Three hundred and one patients receiving partial hepatectomy without any major concomitant surgical procedures, such as bowel or bile duct resection, from May 2016 to June 2017 at the First Affiliated Hospital, School of Medicine, Zhejiang University, China, were included in this study. Data regarding the age, body mass index (BMI), sex, surgical approach, pathological diagnosis, operative time, and intraoperative blood loss were retrospectively collected. Patients were divided into laparoscopic and open hepatectomy groups, and each group was subdivided into an ERAS group, who received the enhanced recovery program, or a control group, who received conventional care.

The ERAS protocol was introduced into our center at January 2017, patients before then were all received conventional care who served as control group, and others were divided into ERAS group. All patients received general anesthesia. Details of the ERAS protocol can be found in the Table S1. In ERAS group, the probiotics was administered only under the consent of patients. All operations were performed by one surgical team including two senior surgeons. Authors had access to information that could identify individual participants during and after data collection. All the patients completed the follow-up and were included in the analysis.

\section{Postoperative evaluation}

Postoperative biochemical parameters, such as total bilirubin (TB), prothrombin time (PT), and aminotransferases were evaluated before the surgery and again 1.0, 3.0, and 30.0 days after surgery. Abdominal distension and pain were evaluated using the numerical rating scale (NRS). Flatus, defecation, and intraperitoneal drainage were assessed daily right after surgery. Time to first flatus and defecation and length of postoperative hospital stay were calculated from surgery to first flatus and defecation or discharge. Data on various complications were collected and classified according to the grading system of Clavien et al. (11). Patients in the ERAS group were then divided in two subgroups, the probiotics group and the non-probiotics group. Patients in the probiotics group received pre- and postoperative probiotics of MIYA-BM ${ }^{\circledR}$ (Miyarisan Pharmaceutical Co., Ltd., Tokyo, Japan), which contains $20.0 \mathrm{mg}$ Clostridium butyricum MIYAIRI (CBM). Two tablets of MIYA-BM were orally administered three times per day. Patients received MIYA-BM for at least 3.0 days before surgery and for 7.0 days after surgery.

\section{Ethical statement}

All patients in the ERAS group were informed of the possible risks before receiving ERAS program. Written informed consent was obtained from all participants. Ethical approval was obtained from the Ethics Committee of the First Affiliated Hospital, School of Medicine, Zhejiang University, China, in accordance with the ethical guidelines 
Table 1 Characteristics and outcomes of patients undergoing hepatectomy

\begin{tabular}{|c|c|c|c|c|c|c|}
\hline Variables & \multicolumn{3}{|c|}{ Laparoscopic hepatectomy ( $\mathrm{n}=182)$} & \multicolumn{3}{|c|}{ Open hepatectomy $(n=119)$} \\
\hline Age, mean $\pm S D$, years & $56.04 \pm 11.50$ & $56.31 \pm 11.57$ & 0.890 & $57.10 \pm 10.42$ & $58.95 \pm 10.89$ & 0.487 \\
\hline $\mathrm{BMI}$, mean $\pm \mathrm{SD}, \mathrm{kg} / \mathrm{m}^{2}$ & $23.70 \pm 3.40$ & $23.54 \pm 3.03$ & 0.768 & $23.09 \pm 3.60$ & $22.74 \pm 3.10$ & 0.652 \\
\hline $\begin{array}{l}\text { Intraoperative blood loss, mean } \\
\pm \mathrm{SD}, \mathrm{mL}\end{array}$ & $167.71 \pm 160.86$ & $132.07 \pm 182.80$ & 0.194 & $160.75 \pm 115.49$ & $199.09 \pm 155.99$ & 0.300 \\
\hline Liver pathology, n (\%) & & & 0.078 & & & 0.341 \\
\hline $\mathrm{cHCC}-\mathrm{CC}$ & $2(4.1)$ & $0(0.0)$ & & $0(0.0)$ & $8(8.1)$ & \\
\hline Benign diseases & $16(32.7)$ & $34(25.6)$ & & $2(10.0)$ & $8(8.1)$ & \\
\hline Resection extent & & & 0.052 & & & 0.132 \\
\hline Minor ( $\leq$ bisegmentectomy) & $35(71.4)$ & $112(84.2)$ & & $13(65.0)$ & $82(82.8)$ & \\
\hline Major (> bisegmentectomy) & $14(28.6)$ & $21(15.8)$ & & $7(35.0)$ & $17(17.2)$ & \\
\hline \multicolumn{7}{|l|}{ Outcomes, mean \pm SD } \\
\hline Time to first flatus, days & $2.25 \pm 0.78$ & $2.68 \pm 0.76$ & 0.002 & $2.90 \pm 0.97$ & $3.46 \pm 1.09$ & 0.035 \\
\hline
\end{tabular}

Benign diseases include hepatic hemangioma, hepatolithiasis, hepatic cyst and others. ERAS, enhanced recovery after surgery; BMI, body mass index; M/F, male/female; POD, postoperative day; CC, cholangiocellular carcinoma; HCC, hepatocellular carcinoma; MHC, metastatic hepatic carcinoma; cHCC-CC, combined hepatocellular carcinoma and cholangiocarcinoma; SD, standard deviation.

of the 1975 Declaration of Helsinki (committee's reference number: 2017-388).

\section{Statistical analyses}

All data were collected retrospectively from patients' medical records. Continuous variables were presented as the mean \pm standard deviation. The Student's $t$-test, chi-squared test, Fisher's exact test, and Kruskal-Wallis $\mathrm{H}$ test were performed accordingly. Variables reaching 10\% significance in univariate analysis were included into multivariate analysis. $\mathrm{P}<0.05$ was considered to be statistically significant. Statistical analyses were performed using SPSS19.0 (SPSS
Inc, Chicago, IL, USA).

\section{Results}

\section{ERAS vs. conventional care in laparoscopic hepatectomy}

A total of 182 patients who underwent laparoscopic hepatectomy were included in the study, of whom 49 were in the ERAS group and 133 were in the control group. Characteristics and outcomes of the patients are provided in Table 1. Age, sex, and BMI were equally distributed among those in the two groups and between the two groups. The operative time for the control group was $189.06 \pm 96.72 \mathrm{~min}$ and for the ERAS group was $247.06 \pm 115.19 \min (\mathrm{P}=0.001)$. 
Table 2 Incidence of complications and complications at different levels of patients undergoing hepatectomy

\begin{tabular}{|c|c|c|c|c|c|c|}
\hline Complications & \multicolumn{3}{|c|}{ Laparoscopic hepatectomy $(\mathrm{n}=182)$} & \multicolumn{3}{|c|}{ Open hepatectomy $(n=119)$} \\
\hline Grade II & $4(8.2)$ & $14(10.5)$ & - & $4(20.0)$ & $26(26.3)$ & - \\
\hline Total & $21(42.9)$ & $84(63.2)$ & 0.014 & $9(45.0)$ & $69(69.7)$ & 0.034 \\
\hline
\end{tabular}

Values are presented as $\mathrm{n}(\%)$. ERAS, enhanced recovery after surgery.

There was no significant difference in intraoperative blood loss between the two groups $(132.07 \pm 182.80 \mathrm{~mL}$ in the control group and $167.71 \pm 160.86 \mathrm{~mL}$ in the ERAS group, $\mathrm{P}=0.194)$. Time to first flatus after surgery was significantly shorter in the ERAS group than in the control group ( $2.25 \pm 0.78$ vs. $2.68 \pm 0.76$ days, respectively; $\mathrm{P}=0.002)$. Length of postoperative hospital stay was also significantly reduced in the ERAS group compared to that in the control group $(6.15 \pm 3.52$ vs. $7.53 \pm 3.77$ days, respectively; $\mathrm{P}=0.029)$. The intraperitoneal drainage 1.0 day after surgery was the same in both groups $(135.89 \pm 227.12 \mathrm{~mL}$ in the control group and $136.30 \pm 185.15 \mathrm{~mL}$ in the ERAS group, $\mathrm{P}=0.991$ ).

In the control group, $63.2 \%$ of the patients had complications compared with only $42.9 \%$ of those in the ERAS group ( $\mathrm{P}=0.014$, Table 2). Although not significantly different $(\mathrm{P}=0.129)$, the complications in ERAS group tended to be milder than those in the control group. The trend in the liver function parameters, including alanine aspartate aminotransferase (AST), alanine aminotransferase (ALT), PT, and TB, were comparable between the two groups (Figure 1).

\section{ERAS vs. traditional care in open hepatectomy}

A total of 119 patients who underwent open hepatectomy were included in this study of which 20 were in the ERAS group and 99 were in the control group. The patient characteristics and the postoperative outcomes of the two groups are provided in Table 2. Patient characteristics were similar regarding age, sex, and BMI. The operative time was $256.95 \pm 107.42 \mathrm{~min}$ for the ERAS group, which was significantly longer than that in the control group $(203.75 \pm 89.03 \mathrm{~min}, \mathrm{P}=0.020)$. No difference was found in intraoperative blood loss between the ERAS and control groups $(160.75 \pm 115.49$ vs. $199.09 \pm 155.99 \mathrm{~mL}$, $\mathrm{P}=0.300$ ). Time to first flatus after surgery was significantly shorter in the ERAS group $(2.90 \pm 0.97$ days $)$ than in the control group $(3.46 \pm 1.09$ days, $\mathrm{P}=0.035)$. The duration of the postoperative hospital stay was $7.33 \pm 1.64$ days in the ERAS group and $12.30 \pm 9.41$ days in the control group $(\mathrm{P}=0.028)$. The intraperitoneal drainage on 1.0 day after surgery was $190.00 \pm 200.50 \mathrm{~mL}$ in the ERAS group and $168.75 \pm 214.62 \mathrm{~mL}$ in the control group $(\mathrm{P}=0.685)$.

Of those in the control group, $69.7 \%$ suffered from complications compared to $45.0 \%$ of patients in the ERAS group ( $\mathrm{P}=0.034$, Table 2). In addition, the complications for those in the ERAS group appeared to be milder; however, no significant difference was found. Changes in liver function in the ERAS and control groups are provided in Figure 2. There was no significant difference between the two groups regarding the AST, ALT, PT, or TB.

\section{Post-operative complications}

In total, 68 patients (22.6\%) suffered grade II-IV complications. Patients with prolonged operative time and more intra-operative blood loss were more likely to had complications more than grade II ( $\mathrm{P}=0.002$ and $\mathrm{P}=0.002$, respectively, Tables 3). Patient sex and liver pathology were found associated with the grade of complications $(\mathrm{P}=0.014$ and $\mathrm{P}=0.033$, respectively, Table 3). The complications in patients received ERAS protocol and laparoscopic hepatectomy tended to be milder $(\mathrm{P}=0.067$ and $\mathrm{P}<0.001$, respectively, Tables 3). After multivariate analysis, the ERAS protocol, laparoscopic hepatectomy and operative time remained to be significant (Tables 4).

\section{Effect of probiotics on patients in the ERAS group}

Patients who underwent laparoscopic hepatectomy in the ERAS group were further divided into two groups 

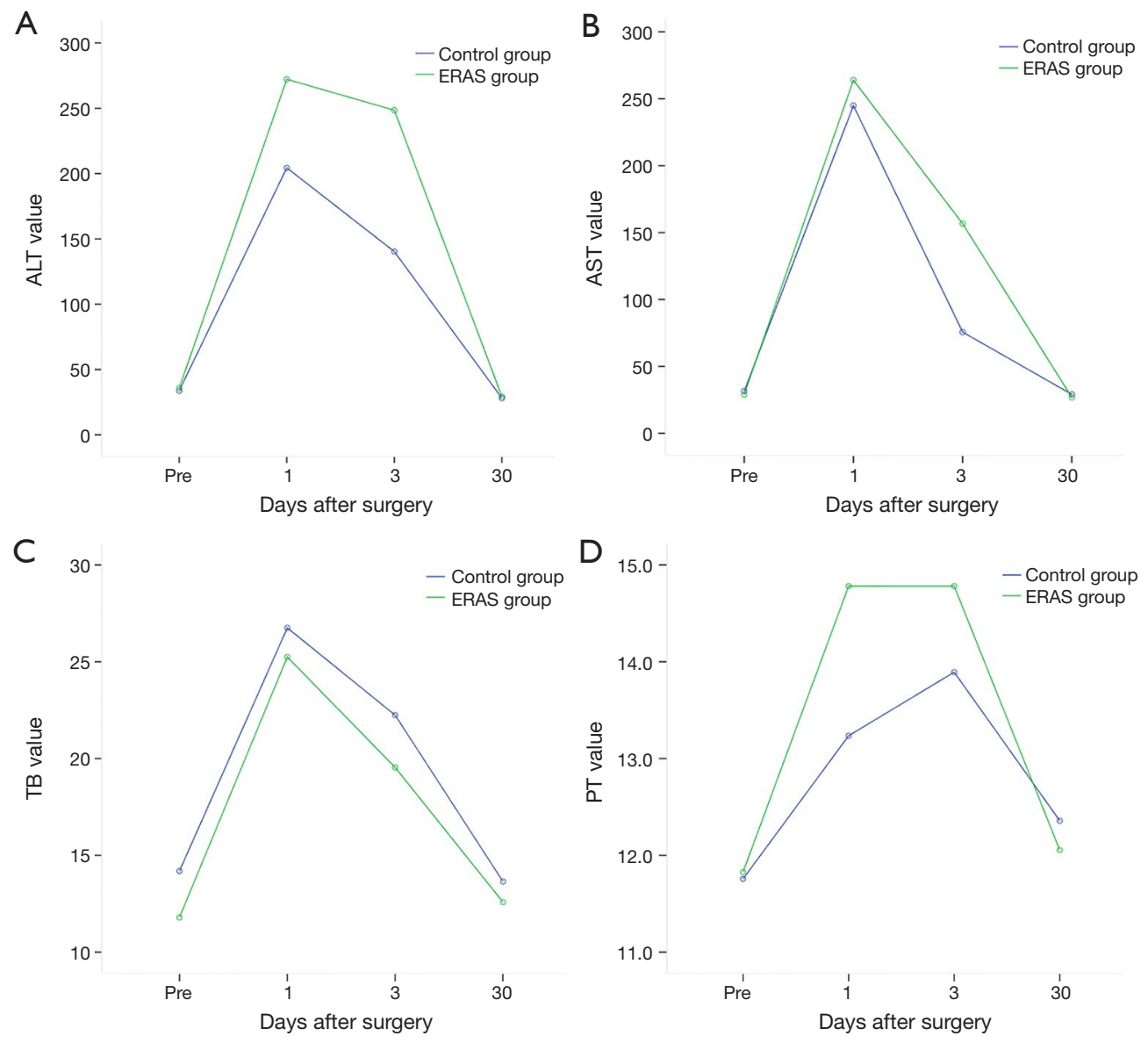

Figure 1 Liver function parameter values before and after laparoscopic hepatectomy. (A) Alanine aminotransferase (ALT), P=0.169; (B) alanine aspartate aminotransferase (AST), $\mathrm{P}=0.418$; (C) total bilirubin (TB), $\mathrm{P}=0.422$; (D) prothrombin time (PT), $\mathrm{P}=0.228$. ERAS, enhanced recovery after surgery.

as follows: 27 in the probiotics group and 22 in the nonprobiotics group. The basic characteristics among them of age, sex, and BMI were not different between the two groups. The intraoperative and postoperative outcomes are provided in Table 5. The time to first flatus after surgery was $2.31 \pm 0.84$ days in the probiotics group and $2.43 \pm 0.93$ days in the non-probiotics group $(\mathrm{P}=0.641)$. The time to first passage of stool after surgery was $3.67 \pm 1.29$ days in the probiotics group and $3.47 \pm 1.18$ days in the nonprobiotics group $(\mathrm{P}=0.657)$. The duration of postoperative hospital stay was $5.63 \pm 3.00$ days in the probiotics group and $7.09 \pm 4.20$ days in the non-probiotics group $(\mathrm{P}=0.162)$. The intraperitoneal drainage 1.0 day after surgery was $99.23 \pm 133.41 \mathrm{~mL}$ in the probiotics group and $184.50 \pm 231.18 \mathrm{~mL}$ in the non-probiotics group $(\mathrm{P}=0.162)$. The abdominal distension and pain score from 1 to 10 based on NRS was decided by the patient on the first post-operative day. The abdominal pain score was $1.00 \pm 1.07$ in the probiotics group and $0.55 \pm 0.80$ in the non-probiotics group $(\mathrm{P}=0.106)$. The abdominal distension score was $0.65 \pm 1.13$ in the probiotics group and $0.55 \pm 0.96$ in the nonprobiotics group $(\mathrm{P}=0.725)$. Similar results were observed in patients receiving open hepatectomy in the ERAS group (Table 5).

\section{Discussion}

This study shows that perioperative management based on an ERAS protocol in hepatectomy patients promoted the recovery of postoperative gastrointestinal function, shortened postoperative hospital stay, and reduced the incidence of postoperative complications. The outcomes were similar in both laparoscopic and open hepatectomy patients. The operative time was longer in the ERAS group 
A
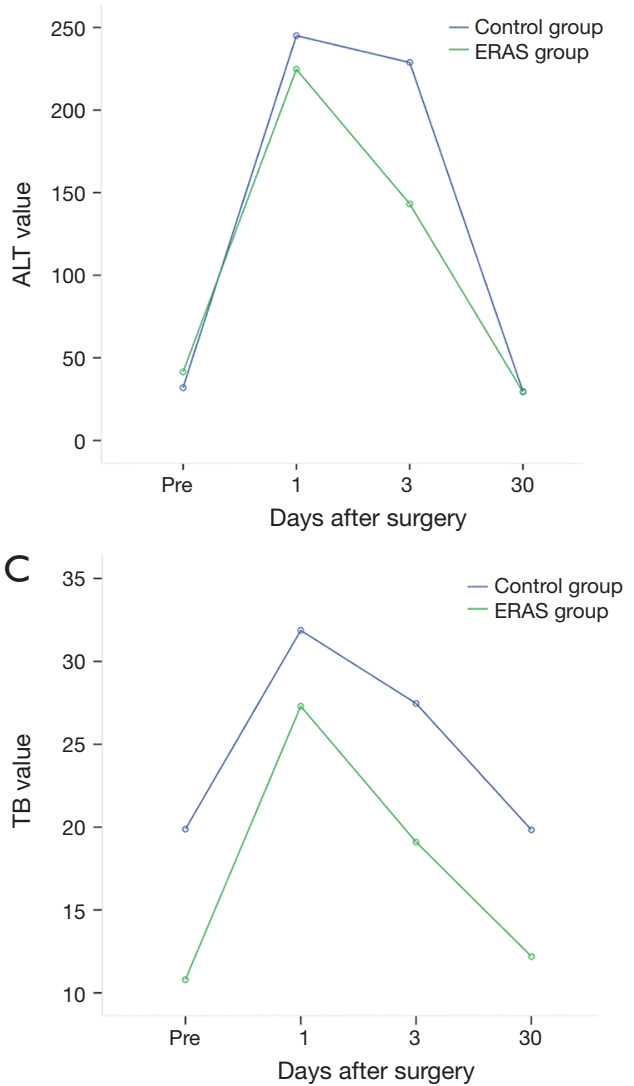

B

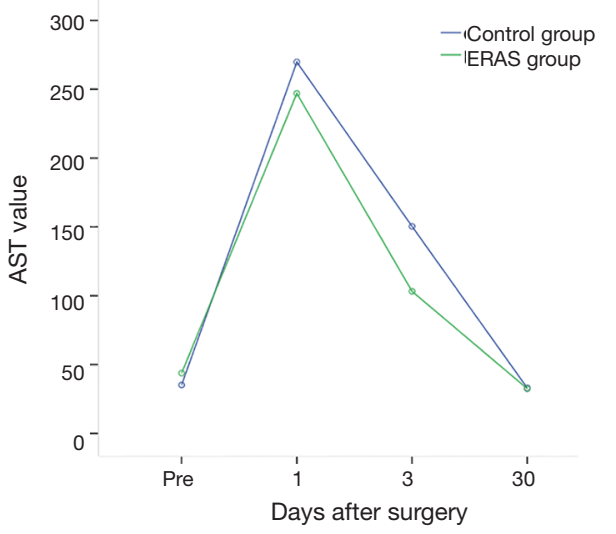

D 18.0

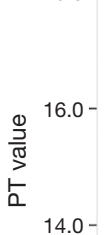

$14.0-$

$12.0-$

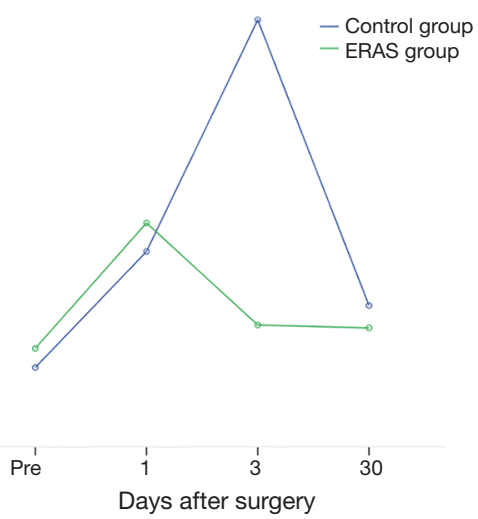

Figure 2 Liver function parameter values before and after open hepatectomy. (A) Alanine aminotransferase (ALT), $\mathrm{P}=0.577$; (B) alanine aspartate aminotransferase (AST), $\mathrm{P}=0.682$; $(\mathrm{C})$ total bilirubin $(\mathrm{TB}), \mathrm{P}=0.716$; (D) prothrombin time (PT), $\mathrm{P}=0.441$. ERAS, enhanced recovery after surgery.

than the control group; however, this was most likely because the ERAS protocol recommended an individualized anesthesia strategy and more meticulous procedures than those used in the control group. The minimally invasive approach recommended in our protocol was a relatively new technique at our institution and as such some of the operating times were longer. We also tried to avoid abdominal drainage tubes after surgery, and so more meticulous hemostasis was required. Both these factors contributed to difference in the length of operation between groups. Nonetheless, the results showed that even the prolonged operative time could increase the incidence of post-operative complications, however, the overall outcomes were more favorable in the ERAS group. Subgroup analysis of the results of the use of probiotics in the ERAS group revealed that short-term perioperative intake of probiotics did not promote the early return of bowel functions or improve the prognosis after surgery.
It has been proven that routine nasogastric tube use could increase pulmonary complications and extend the recovery time for intestinal function (12). In this study, the nasogastric tube was not routinely used but, if used, was removed immediately after surgery in the ERAS group. A previous report emphasized the safety and benefits of early oral intake of nourishment without increasing complications or mortality (13). Early oral intake of nourishment was suggested for patients as part of the ERAS protocol. The patients were allowed to have carbohydrate drinks without residue $6 \mathrm{~h}$ after surgery. If there was no nausea or vomiting, the patients were allowed to have solid food on the first postoperative day. Most patients could eat normal food on the post-operative day 2 . No complications related to early oral intake was observed in the present study. Minimally invasive approach was recommended for ERAS protocol, and the results in present study confirmed that patients receiving laparoscopic hepatectomy had fewer complications than 
Table 3 Comparison between patients underwent post-operative grade 0-I and grade II-IV complications

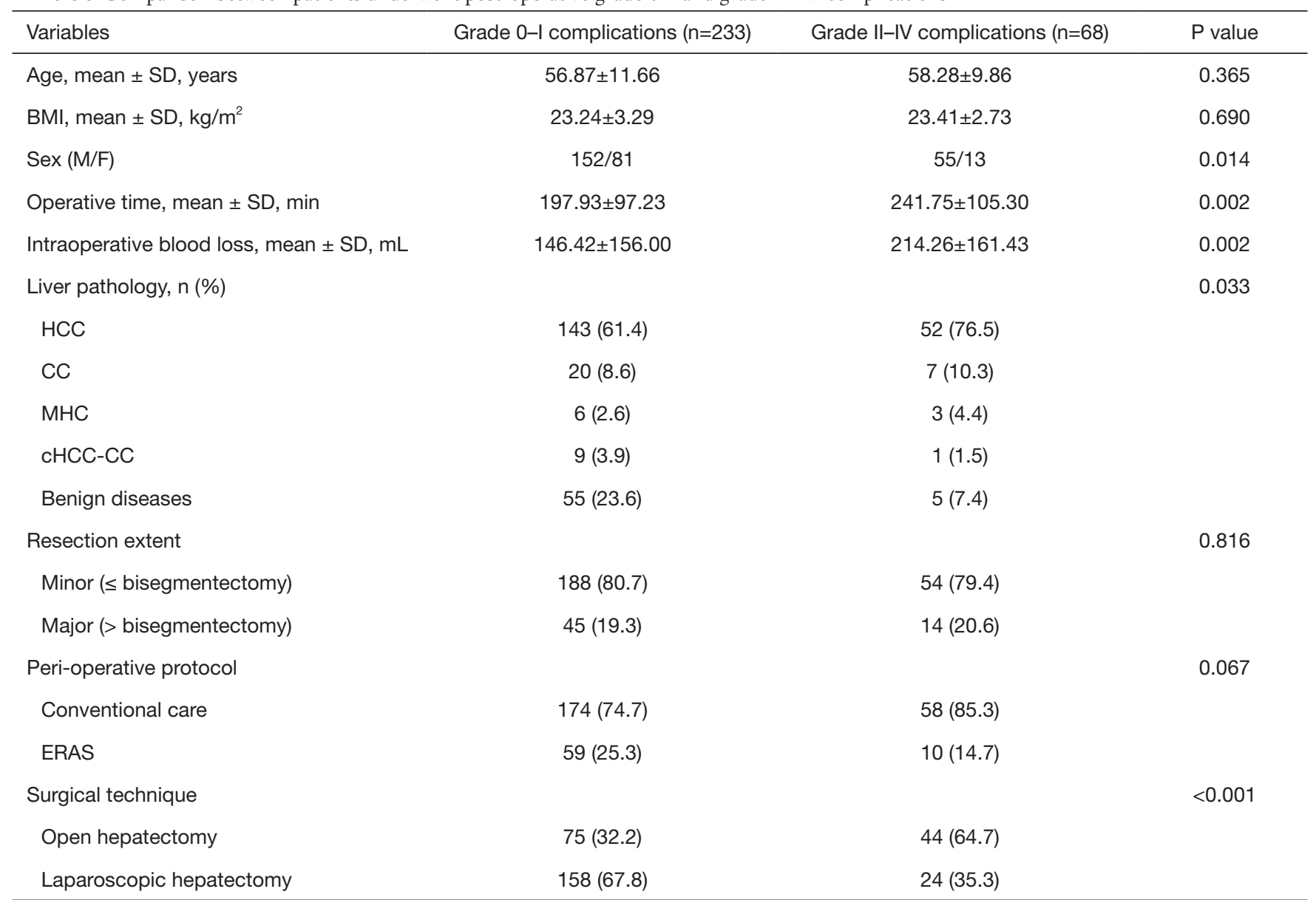

Benign diseases include hepatic hemangioma, hepatolithiasis, hepatic cyst and others. ERAS, enhanced recovery after surgery; BMI, body mass index; M/F, male/female; POD, postoperative day; CC, cholangiocellular carcinoma; HCC, hepatocellular carcinoma; MHC, metastatic hepatic carcinoma; $\mathrm{CHCC}-\mathrm{CC}$, combined hepatocellular carcinoma and cholangiocarcinoma; SD, standard deviation.

Table 4 Multivariate analysis for post-operative complications

\begin{tabular}{lccc}
\hline Variables & $\begin{array}{c}\text { Hazard } \\
\text { ratio }\end{array}$ & $\begin{array}{c}95 \% \text { confidence } \\
\text { interval }\end{array}$ & P value \\
\hline $\begin{array}{l}\text { Peri-operative protocol } \\
\text { Conventional care }\end{array}$ & 1.000 & - & - \\
ERAS & 0.376 & $0.163-0.868$ & 0.022 \\
$\begin{array}{l}\text { Surgical technique } \\
\text { Open hepatectomy }\end{array}$ & 1.000 & - & - \\
$\begin{array}{l}\text { Laparoscopic } \\
\text { hepatectomy }\end{array}$ & 0.258 & $0.143-0.468$ & $<0.001$ \\
Operative time, min & 1.005 & $1.002-1.008$ & $<0.001$ \\
\hline
\end{tabular}

ERAS, enhanced recovery after surgery. those receiving open hepatectomy and recovered fasted after surgery. Therefore, the laparoscopic hepatectomy should always be the first choice when it was possible.

Evidence for the recommendation of early mobilization after surgery as part of the ERAS protocol was limited (14); however, it is clear that extended bed rest is associated with multiple deleterious effects, such as diffuse muscle atrophy, thromboembolic disease, and insulin resistance $(15,16)$. In our ERAS protocol, we encouraged early mobilization. On the day of surgery, few hours after surgery completed, patients in the ERAS group were recommended bed exercises under the guidance of rehabilitation physicians. One day after surgery, off-bed activity was suggested with help from the 
Table 5 Characteristics and outcomes of patients in ERAS group

\begin{tabular}{|c|c|c|c|c|c|c|}
\hline Variables & \multicolumn{3}{|c|}{ Laparoscopic hepatectomy $(\mathrm{n}=49)$} & \multicolumn{3}{|c|}{ Open hepatectomy $(n=20)$} \\
\hline $\mathrm{BMI}$, mean $\pm \mathrm{SD}, \mathrm{kg} / \mathrm{m}^{2}$ & $23.31 \pm 2.87$ & $24.18 \pm 3.98$ & 0.380 & $21.75 \pm 2.87$ & $24.74 \pm 3.86$ & 0.062 \\
\hline $\begin{array}{l}\text { Intraoperative blood loss, } \\
\text { mean } \pm \mathrm{SD}, \mathrm{mL}\end{array}$ & $140.00 \pm 123.03$ & $203.33 \pm 196.88$ & 0.179 & $174.09 \pm 136.69$ & $144.44 \pm 88.19$ & 0.582 \\
\hline $\begin{array}{l}\text { Time to first flatus, mean } \pm \\
S D \text {, days }\end{array}$ & $2.31 \pm 0.84$ & $2.43 \pm 0.93$ & 0.641 & $2.73 \pm 1.10$ & $3.11 \pm 0.78$ & 0.392 \\
\hline $\begin{array}{l}\text { Time to off-bed activity, } \\
\text { mean } \pm S D \text {, days }\end{array}$ & $2.62 \pm 1.39$ & $2.68 \pm 1.09$ & 0.856 & $3.00 \pm 1.10$ & $2.33 \pm 0.87$ & 0.155 \\
\hline $\begin{array}{l}\text { Postoperative hospital stay, } \\
\text { mean } \pm S D \text {, days }\end{array}$ & $5.63 \pm 3.00$ & $7.09 \pm 4.20$ & 0.162 & $8.55 \pm 3.30$ & $8.11 \pm 3.92$ & 0.795 \\
\hline Pain score, mean \pm SD & $1.00 \pm 1.07$ & $0.55 \pm 0.80$ & 0.106 & $0.91 \pm 1.22$ & $0.67 \pm 1.00$ & 0.628 \\
\hline $\begin{array}{l}\text { Abdominal distension } \\
\text { score, mean } \pm S D\end{array}$ & $0.65 \pm 1.13$ & $0.55 \pm 0.96$ & 0.725 & $0.36 \pm 0.81$ & $0.89 \pm 1.45$ & 0.32 \\
\hline $\begin{array}{l}\text { Intraperitoneal drainage on } \\
\mathrm{POD} 1, \text { mean } \pm \mathrm{SD}, \mathrm{mL}\end{array}$ & $99.23 \pm 133.41$ & $184.50 \pm 231.18$ & 0.162 & $187.27 \pm 150.14$ & $193.33 \pm 259.37$ & 0.95 \\
\hline
\end{tabular}

ERAS, enhanced recovery after surgery; BMI, body mass index; M/F, male/female; POD, postoperative day; SD, standard deviation.

nurses. Pain control helped to promote early off-bed activity. Although evidence is lacking to prove the effectiveness of early mobilization, we observed daily postoperative progress from patient activity, such as decreased first time to flatus and defecation and decreased hospital stay.

Probiotics are live bacteria that might provide health benefits when consumed. These beneficial bacteria can prevent harmful bacterial infections by inhibiting bacterial overgrowth and translocation. In addition, probiotics might enhance immune system function when used as a dietary supplement, presumably by activating systemic cellular immune responses and boosting natural immunity (17). The perioperative application of probiotics was reported to improve the postoperative recovery of peristalsis and decrease infectious complications in patients with colorectal cancer undergoing colectomy (18). However, Gurusamy et al. (19) reviewed the methods of preventing bacterial sepsis and wound complications after liver transplantation and revealed that there is no clear evidence to support that probiotics decreased infections. In our study, a subgroup analysis was conducted to determine whether short-term use of probiotics during the perioperative period could improve the outcomes in patients in the ERAS group. Our results showed no difference in postoperative outcomes between the patients who received perioperative probiotics and those who did not. This was a pilot study and so the noneffectiveness of the probiotics might have been because of the small sample size of the groups and the short timeframe for probiotics consumption. Further studies based on a large and sufficient cohort based on power calculation and extended timeframe for consumption of probiotics are needed to confirm the results. 


\section{Conclusions}

In conclusion, the ERAS protocol for hepatectomy patients could help promote the recovery of gastrointestinal function, shorten postoperative hospital stay, and reduce the incidence of postoperative complications. Perioperative short-term probiotics use based on the ERAS protocol did not improve postoperative outcomes. Nevertheless, the present study has some limitations. It was a single center retrospective analysis with a lack of homogeneity. A randomized, large-sample, multi-center research should be conducted in the future.

\section{Acknowledgments}

Funding: This work was supported by National Natural Science Foundation of China (No.81372626, No.81572975), Key Research and Development Project of Science and Technology Department of Zhejiang, China (No.2015C03053), and Zhejiang Provincial Program for the Cultivation of High-level Innovative Health Talents [2016].

\section{Footnote}

Conflicts of Interest: All authors have completed the ICMJE uniform disclosure form (available at http://dx.doi. org/10.21037/tcr.2018.08.30). The authors have no conflicts of interest to declare.

Ethical Statement: The authors are accountable for all aspects of the work in ensuring that questions related to the accuracy or integrity of any part of the work are appropriately investigated and resolved. Ethical approval was obtained from the Ethics Committee of the First Affiliated Hospital, School of Medicine, Zhejiang University, China, in accordance with the ethical guidelines of the 1975 Declaration of Helsinki (committee's reference number: 2017-388). Written informed consent was obtained from all participants.

Open Access Statement: This is an Open Access article distributed in accordance with the Creative Commons Attribution-NonCommercial-NoDerivs 4.0 International License (CC BY-NC-ND 4.0), which permits the noncommercial replication and distribution of the article with the strict proviso that no changes or edits are made and the original work is properly cited (including links to both the formal publication through the relevant DOI and the license).
See: https://creativecommons.org/licenses/by-nc-nd/4.0/.

\section{References}

1. Wilmore DW, Kehlet H. Management of patients in fast track surgery. BMJ 2001;322:473-6.

2. Kehlet H. Multimodal approach to control postoperative pathophysiology and rehabilitation. Br J Anaesth 1997;78:606-17.

3. Nicholson A, Lowe MC, Parker J, et al. Systematic review and meta-analysis of enhanced recovery programmes in surgical patients. Br J Surg 2014;101:172-88.

4. Teeuwen PH, Bleichrodt RP, Strik C, et al. Enhanced recovery after surgery (ERAS) versus conventional postoperative care in colorectal surgery. J Gastrointest Surg 2010;14:88-95.

5. Gatenby PA, Shaw C, Hine C, et al. Retrospective cohort study of an enhanced recovery programme in oesophageal and gastric cancer surgery. Ann R Coll Surg Engl 2015;97:502-7.

6. Arumainayagam N, McGrath J, Jefferson KP, et al. Introduction of an enhanced recovery protocol for radical cystectomy. BJU Int 2008;101:698-701.

7. Koo V, Brace H, Shahzad A, et al. The challenges of implementing Enhanced Recovery Programme in urology. Int J Urol Nurs 2013;7:106-10.

8. Nelson G, Kalogera E, Dowdy SC. Enhanced recovery pathways in gynecologic oncology. Gynecol Oncol 2014;135:586-94.

9. Song W, Wang K, Zhang RJ, et al. The enhanced recovery after surgery (ERAS) program in liver surgery: a metaanalysis of randomized controlled trials. Springerplus 2016;5:207.

10. Wallström A, Frisman GH. Facilitating early recovery of bowel motility after colorectal surgery: a systematic review. J Clin Nurs 2014;23:24-44.

11. Dindo D, Demartines N, Clavien PA. Classification of surgical complications: a new proposal with evaluation in a cohort of 6336 patients and results of a survey. Ann Surg 2004;240:205-13.

12. Nelson R, Edwards S, Tse B. Prophylactic nasogastric decompression after abdominal surgery. John Wiley \& Sons, Ltd., 2004.

13. Lewis SJ, Egger M, Sylvester PA, et al. Early enteral feeding versus "nil by mouth" after gastrointestinal surgery: systematic review and meta-analysis of controlled trials. BMJ 2001;323:773-6.

14. Melloul E, Hübner M, Scott M, et al. Guidelines for 
Perioperative Care for Liver Surgery: Enhanced Recovery After Surgery (ERAS) Society Recommendations. World J Surg 2016;40:2425-40.

15. Kehlet $\mathrm{H}$, Wilmore DW. Multimodal strategies to improve surgical outcome. Am J Surg 2002;183:630-41.

16. Brower RG. Consequences of bed rest. Crit Care Med 2009;37:S422-8.

17. Sheih YH, Chiang BL, Wang LH, et al. Systemic immunity-enhancing effects in healthy subjects following dietary consumption of the lactic acid bacterium

Cite this article as: Ding Y, Gao Z, Sun Z, Zhang Q, Zhou B, Li Z, Zhang W, Jiang Y, Zhang X, Ge G, Ravaioli M, Jones C, Yan S, Wang W; written on behalf of the AME Liver Surgery Collaborative Group. Enhanced recovery program in liver resection surgery: a single center experience. Transl Cancer Res 2018;7(4):1112-1121. doi: 10.21037/tcr.2018.08.30
Lactobacillus rhamnosus HN001. J Am Coll Nutr. 2001;20:149-56.

18. Liu Z, Qin H, Yang Z, et al. Randomised clinical trial: the effects of perioperative probiotic treatment on barrier function and post-operative infectious complications in colorectal cancer surgery - a double-blind study. Aliment Pharmacol Ther 2011;33:50-63.

19. Gurusamy KS, Kumar Y, Davidson BR. Methods of preventing bacterial sepsis and wound complications for liver transplantation. John Wiley \& Sons, Ltd., 2008. 
Table S1 Details of the ERAS protocol

\begin{tabular}{|c|c|}
\hline ERAS items & Summary \\
\hline \multicolumn{2}{|l|}{ Day before surgery } \\
\hline Preoperative counseling & Patients would receive routine preoperative counseling and education about ER \\
\hline Preoperative exercise & $\begin{array}{l}\text { Patients were suggested to walk } 5,000-10,000 \text { steps everyday (or other ways to } \\
\text { the same amount of exercise) }\end{array}$ \\
\hline Preoperative diets & Patients were recommended to eat low-fat, fresh food and high-quality protein \\
\hline Preoperative nutrition & $\begin{array}{l}\text { Patients with at least one of the following criteria: weight loss }>10-15 \% \text { within } \\
6 \text { months, BMI }<18.5 \mathrm{~kg} / \mathrm{m}^{2} \text { and serum albumin }<30 \mathrm{~g} / \mathrm{L} \text { without hepatic or renal } \\
\text { dysfunction were recommended } 5-7 \text { days of oral supplements before surgery }\end{array}$ \\
\hline
\end{tabular}

No fasting more than $6 \mathrm{~h}$ before surgery

Ensure adequate sleep

\section{Day of surgery}

Minimally invasive approach

No nasogastric tube

Early exercise on bed

Take a deep breath and cough exercise

Early oral intake

POD 1

Abdominal pain evaluation
Off-bed activity

Continuous breathing exercise

Temporary eating restrictions

Venous fluid reduction

POD 2

Abdominal pain evaluation

Continuous breathing exercise

Off-bed activity

Liquids and Solid food intake

POD 3

Abdominal pain evaluation
Continuous breathing exercise
Off-bed activity

Off-bed activity

Liquids and solid food intake

Discharge assessment

POD 4

Continuous exercise

Discharge assessment
Preoperative fasting was required no more than $2 \mathrm{~h}$ for liquids and $6 \mathrm{~h}$ for solid food If patients complained of sleep problems, proper medications would be provided to ensure adequate sleep

\section{Laparoscopic surgery was first recommended}

Nasogastric tube was not routinely placed. If nasogastric tube was used during the surgery, it would be removed immediately after surgery

Patients were recommended to do some early lower-limb exercise on bed under the guidance of rehabilitation physicians to improve blood circulation (repeated 4-5 times every half an hour during awake)

Patients should use breathing exerciser or balloon to do breathe exercise at least 10 times per hour during awake. A cough was performed at the end of each breach exercise

Patients were suggested to have some water, rice soup without rice grain and some drinks without residue $6 \mathrm{~h}$ after surgery

Abdominal pain was evaluated by NRS. Pain control must be adequate

The catheter was removed in the early morning and patients would do intermittent off-bed exercise for at least $2 \mathrm{~h}$ (mainly bedside activities)

\section{As described before}

Patients continued to take liquids and protein drinks per os during the first postoperative day. Solid food could be added if the reaction was good. Antiemetic could be adopted. Parenteral feeding would be reserved for malnourished patients or those with prolonged fasting due to complications (such as delayed gastric emptying)

According the fluid patients drank, venous fluid reduction would be reduced

As described before

As described before

Patients would do intermittent off-bed exercise for at least $4 \mathrm{~h}$ (walked at least twice, each time no less than 10 meters)

Temporary eating restrictions were still suggested and patients could take liquids and some solid food

\section{As described before}

As described before

Patients would do intermittent off-bed exercise for at least $6 \mathrm{~h}$ (walked at least triple, each time no less than 20 meters)

\section{As described before}

Patients could prepare for discharge in the next day when the peritoneal drainage fluid was clear and less than $50 \mathrm{~mL}$ per day, and patients could get out of bed for daily activities

Patients should increase the amount of exercise according to their own status As described before

ERAS, enhanced recovery after surgery; BMI, body mass index; NRS, numerical rating scale; POD, postoperative day. 